AIP Applied Physics

\title{
Approaching intrinsic performance in ultra-thin silicon nitride drum resonators
}

\author{
V. P. Adiga, B. Ilic, R. A. Barton, I. Wilson-Rae, H. G. Craighead et al.
}

Citation: J. Appl. Phys. 112, 064323 (2012); doi: 10.1063/1.4754576

View online: http://dx.doi.org/10.1063/1.4754576

View Table of Contents: http://jap.aip.org/resource/1/JAPIAU/v112/i6

Published by the American Institute of Physics.

\section{Related Articles}

Ultra-sensitive magnetoelectric microcantilever at a low frequency Appl. Phys. Lett. 101, 182902 (2012)

Two orders of magnitude increase in metal piezoresistor sensitivity through nanoscale inhomogenization J. Appl. Phys. 112, 084332 (2012)

Gold coating of micromechanical DNA biosensors by pulsed laser deposition

J. Appl. Phys. 112, 084330 (2012)

Hand-powered microfluidics: A membrane pump with a patient-to-chip syringe interface Biomicrofluidics 6, 044102 (2012)

High quality factor single-crystal diamond mechanical resonators

Appl. Phys. Lett. 101, 163505 (2012)

\section{Additional information on J. Appl. Phys.}

Journal Homepage: http://jap.aip.org/

Journal Information: http://jap.aip.org/about/about_the_journal

Top downloads: http://jap.aip.org/features/most_downloaded

Information for Authors: http://jap.aip.org/authors

\section{ADVERTISEMENT}
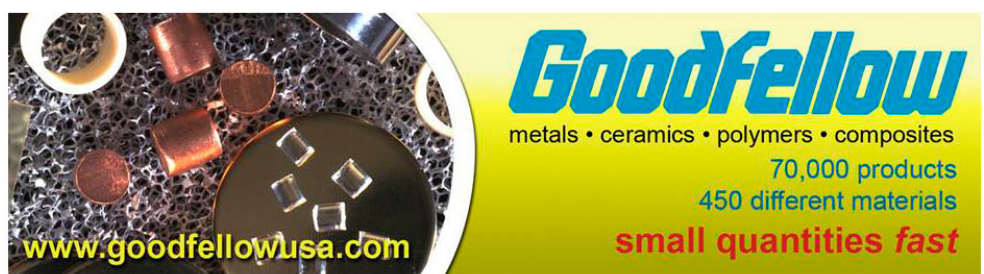


\title{
Approaching intrinsic performance in ultra-thin silicon nitride drum resonators
}

\author{
V. P. Adiga, ${ }^{1, a)}$ B. Ilic, ${ }^{2}$ R. A. Barton, ${ }^{1}$ I. Wilson-Rae,${ }^{3,4}$ H. G. Craighead,${ }^{1}$ and J. M. Parpia ${ }^{5}$ \\ ${ }^{1}$ School of Applied and Engineering Physics, Cornell University, Ithaca, New York 14853, USA \\ ${ }^{2}$ Cornell Nanofabrication facility, Cornell University, Ithaca, New York 14853, USA \\ ${ }^{3}$ Institute for Theoretical Physics, Universität Erlangen-Nürnberg, Erlangen, Germany \\ ${ }^{4}$ Department of Physics, Technische Universität, München, Garching, Germany \\ ${ }^{5}$ Department of Physics, Cornell University, Ithaca, New York 14853, USA
}

(Received 8 May 2012; accepted 24 August 2012; published online 26 September 2012)

\begin{abstract}
We have fabricated circular silicon nitride drums of varying diameter (20 $\mu \mathrm{m}$ to $1 \mathrm{~mm})$ and thickness $(15 \mathrm{~nm}-75 \mathrm{~nm})$ using electron beam lithography and measured the dissipation $\left(\mathrm{Q}^{-1}\right)$ of these amorphous silicon nitride resonators using optical interferometric detection. We observe that the dissipation is strongly dependent on mode type for relatively large, thick membranes as predicted by the current models of dissipation due to clamping loss. However, this dependence is drastically reduced for smaller or thinner resonators, with thinner resonators showing higher quality factors, for low order modes. Highest quality factors that can be reached for these thin resonators seems be limited by an intrinsic mechanism and scales linearly with the diameter of the membrane. Our results are promising for mass sensing and optomechanical applications where low mass and high Qs are desirable. (C) 2012 American Institute of Physics. [http://dx.doi.org/10.1063/1.4754576]
\end{abstract}

\section{INTRODUCTION}

Stoichiometric high tensile stress $(\sim 1.0 \mathrm{GPa})$ silicon nitride membranes and string resonators have shown extremely high mechanical quality factors $(\mathrm{Q}>1000000)$ at room temperature ${ }^{1-6}$ and are useful for force and mass sensing experiments. ${ }^{2,7,8}$ These silicon nitride membranes are being increasingly used in high finesse optical cavities where their low spring constant and high mechanical quality factors prove ideal for realizing dispersive optomechanical experiments. ${ }^{2,5,8}$ In this context, using very large, ultra-light membranes as the mechanical components is advantageous to achieve low spring constants and resonator masses. ${ }^{2}$ However, achieving large, thin, suspended devices with high $Q$ in these high surface-to-volume-ratio $(\mathrm{R})$ resonators has been a challenge. ${ }^{9,10}$ Recently it has been observed that the Qfactors of these resonators can be significantly improved by choosing appropriate resonator geometries, mode shapes, and optimized fabrication techniques. ${ }^{11,12}$ In this article, we present the measured quality factor of e-beam defined thin circular silicon nitride membranes as a function of diameter, thickness, and mode shape. The observed mechanical $Q$ shows a strong modal dependence for large resonators made from thicker films, due to the clamping loss; for thinner films or smaller resonators the dissipation $\left(\mathrm{Q}^{-1}\right)$ of all the modes is dominated instead by an intrinsic dissipation mechanism which is weakly dependent on mode number of the resonator. For comparable dimensions (Diameter, D $>200 \mu \mathrm{m}$ ) and given mode type, thinner resonators always show a higher $\mathrm{RQ}$ product (surface to volume ratio $(\mathrm{R})$ times quality factor (Q)). Thus for a certain diameter $(700 \mu \mathrm{m}) \mathrm{Q}$ values of up to $4.4 \times 10^{6}$ can be achieved with very thin $(15 \mathrm{~nm})$ membranes despite the increase in the surface-to-volume ratio. As we will

\footnotetext{
${ }^{\text {a) }}$ Author to whom correspondence should be addressed. Electronic mail: vpa8@cornell.edu.
}

discuss, in most resonators having a nanoscale thickness, Q varies inversely with the thickness, a trend that is violated by these high stress nitride films. This provides insight into the dissipation mechanisms in high stress nitride films, and thus provides a means to find optimum geometries and mode shapes for achieving high frequency high-Q resonators with low mass.

\section{FABRICATION OF DEVICES AND EXPERIMENTAL METHODS}

Stoichiometric silicon nitride films with a tensile stress ( $\sigma \sim 1.0 \mathrm{GPa})$ were deposited on top of $660 \mathrm{~nm}$ of thermal oxide on a silicon substrate using low pressure chemical vapor deposition (LPCVD) process. To achieve uniform thickness, well-defined release holes and resonator boundaries we fabricated circular membranes (drums) of diameter between $20 \mu \mathrm{m}$ and $1 \mathrm{~mm}$ by generating a pattern of circularly symmetric $100 \mathrm{~nm}$ diameter etch release holes spaced $1 \mu \mathrm{m}$ apart with electron beam (e-beam, GEOL 9300) lithography (Figure 1). The pattern was generated on high stress silicon nitride films of initial thickness $110 \mathrm{~nm}$ and $55 \mathrm{~nm}$. These e-beam defined resonators were released using a timed hydrofluoric acid (49\%) etch followed by critical point drying, leading to more uniform cross sections (Figure 1(d)) than what we measured in resonators fabricated using optical lithography where etch holes were spaced $5 \mu \mathrm{m}$ apart. ${ }^{11}$ In those resonators, the longer etch time required to release the resonator from the underlying substrate partially consumed much of the silicon nitride, resulting in a much deeper "honeycomb" pattern. Resonators with thicknesses ranging from $15 \mathrm{~nm}$ to $75 \mathrm{~nm}$ were fabricated using the e-beam defined process. Thicknesses were measured after peeling off the resonator from the supporting nitride using polydimethylsiloxane (PDMS) and imaging the back plane with atomic force microscopy (AFM), as shown in Figure 1(d). For thinner resonators, thicknesses were estimated using etch 

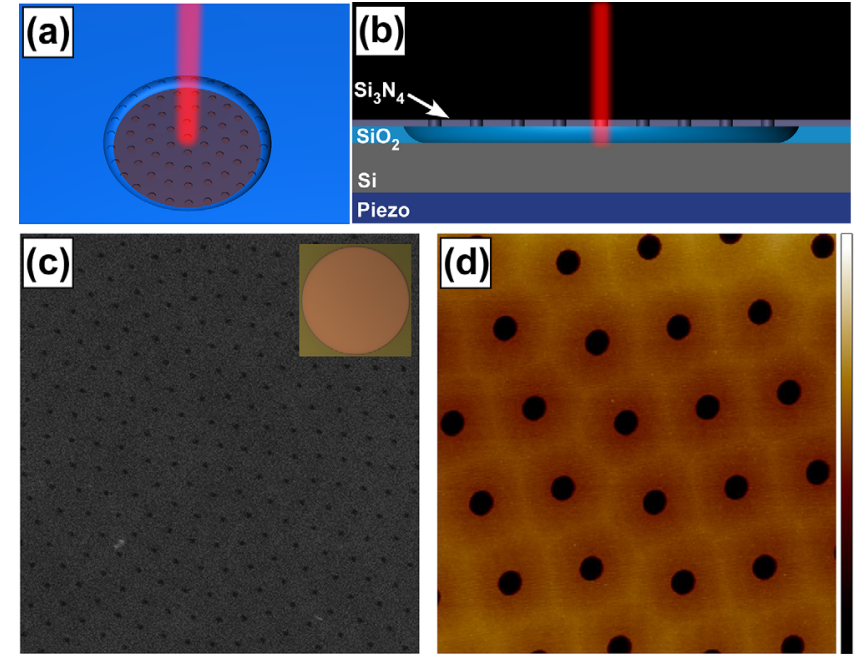

FIG. 1. (a) Schematic of circular membrane with e-beam defined perforations along with a representation of the detection laser beam $(\mathrm{HeNe})(\mathrm{b})$ Schematic of cross section of the device with piezo actuation and detection laser beam (c) SEM image of the central portion of the suspended membrane resonator showing circular symmetric $100 \mathrm{~nm}$ etch release holes that are spaced $1 \mu \mathrm{m}$ apart. Inset: Optical image of high stress silicon nitride membrane (300 $\mu \mathrm{m}$ diameter). (d) AFM image $(5 \mu \mathrm{m} \times 5 \mu \mathrm{m})$ of the backside of the released membrane with uniform cross section (color scale $0-40 \mathrm{~nm}$ ). Released membranes are peeled off using PDMS before imaging using AFM to determine the uniformity and thickness of the membranes.

rates of the nitride from its initial thickness $(55 \mathrm{~nm})$. Resonance frequencies and $\mathrm{Q}$ factors of different modes were measured interferometrically ${ }^{13}$ with piezoelectric actuation under high vacuum conditions $\left(<2 \times 10^{-6}\right.$ Torr $)$.

\section{RESULTS AND DISCUSSION}

The resonant frequencies $\omega_{\mathrm{n}, \mathrm{m}}=2 \pi \mathrm{f}_{\mathrm{n}, \mathrm{m}}$ of tensile circular membranes with diameter (D) are given by ${ }^{14}$

$$
\omega_{n, m}=2 \xi_{n, m} \frac{c_{R}}{D} .
$$

The different types of modes are labeled by $\mathrm{n}=0,1,2, \ldots$, and $\mathrm{m}=1,2,3, \ldots$, where $\mathrm{n}$ is the azimuthal mode number (corresponding to "cake-like-modes") and $\mathrm{m}$ is the radial mode number (indicating the number of radial nodal lines starting with $\mathrm{m}=1$ at the periphery of the resonator). $c_{R}=\sqrt{\sigma / \rho}$ is the phase velocity ( $\rho$ and $\sigma$ are, respectively, the density and tensile stress) and $\xi_{n, m}$ is the $\mathrm{m}^{\text {th }}$ zero of Bessel function $\mathrm{J}_{\mathrm{n}}$ (x). Figure 2 shows the resonant frequencies as a function of wavenumber $\left(\mathrm{k}_{\mathrm{n}, \mathrm{m}}=2 \xi_{\mathrm{n}, \mathrm{m}} / \mathrm{D}\right)$ for all the modes of resonators with diameters ranging from $20 \mu \mathrm{m}$ to $1 \mathrm{~mm}$ and two different thicknesses. As expected the resonant frequencies show an inverse relationship with diameter, yielding phase velocities of $455 \mathrm{~m} / \mathrm{s}$ and $587 \mathrm{~m} / \mathrm{s}$ for $15 \mathrm{~nm}$ (initial thickness, $55 \mathrm{~nm}$ ) and $75 \mathrm{~nm}$ (initial thickness, $110 \mathrm{~nm}$ ) thick films, respectively (after fitting harmonics for all diameters for a given thickness.) This indicates an average stress of $560 \mathrm{MPa}$ for $15 \mathrm{~nm}$ films and $930 \mathrm{MPa}$ for $75 \mathrm{~nm}$ resonators. Welldefined resonator boundaries, etch holes, and uniform thicknesses of the resonators made via e-beam lithography result in frequencies that are in excellent agreement with tautmembrane theory, with deviations of calculated modes from

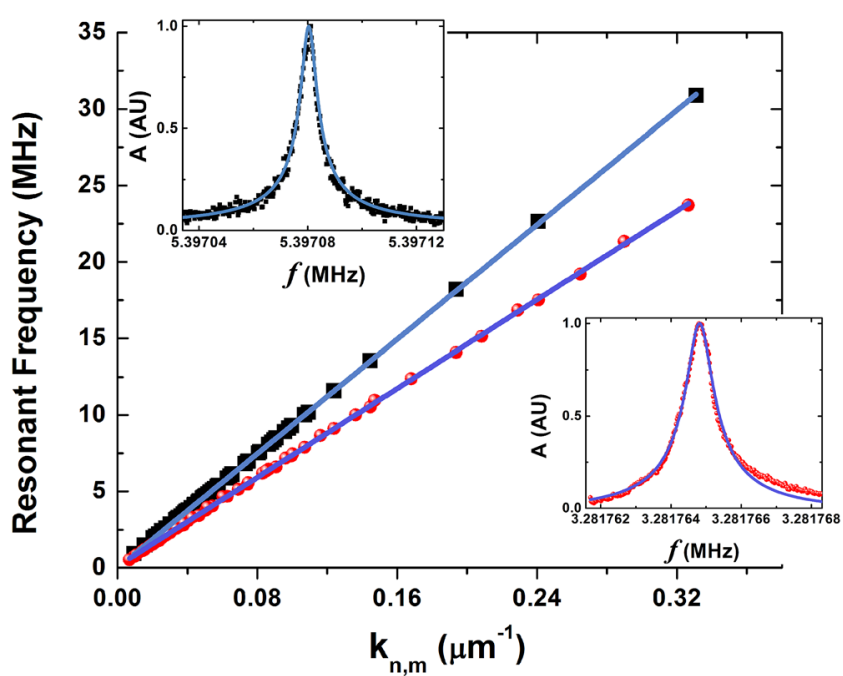

FIG. 2. Resonant frequency as a function of wavenumber $\left(\mathrm{k}_{\mathrm{nm}}\right)$ for resonators with diameters ranging from $20 \mu \mathrm{m}$ to $1 \mathrm{~mm}$. Included in this plot are all the modes measured for individual resonators. Black squares, and red circles indicate, respectively, $75 \mathrm{~nm}$ and $15 \mathrm{~nm}$ thick films. Fits yield a phase velocities $587 \mathrm{~m} / \mathrm{s}$ and $455 \mathrm{~m} / \mathrm{s}$, respectively, with less than $0.1 \%$ deviation indicating very well defined resonator geometries. Insets: Lorentzian fits to an azimuthal mode $(\mathrm{Q} \sim 1000000, \mathrm{n}=3, \mathrm{~m}=1, \mathrm{D}=300 \mu \mathrm{m}, \mathrm{t}=75 \mathrm{~nm}$, black squares) and a pure radial mode $(\mathrm{Q} \sim 4400000, \mathrm{n}=0, \mathrm{~m}=5, \mathrm{D}=700 \mu \mathrm{m}$, $\mathrm{t}=15 \mathrm{~nm}$, red circles).

their measured values of less than $0.1 \%$ (Figure 2). Similarly, as a result of the well-defined resonator boundaries shown in Figure 1, we generally do not observe splitting of the modal degeneracy of the higher order $(n>0)$ azimuthal modes due to geometrical asymmetry.

Figure 3 shows the measured dissipation $\left(\mathrm{Q}^{-1}\right)$ of the fundamental radial mode $(\mathrm{n}=0, \mathrm{~m}=1)$ and its azimuthal harmonics $(n=0,1,2, \ldots, m=1)$ of resonators with different diameters. The dissipation as a function of resonant frequency within a given "family" $(\mathrm{n}=0,1,2,3, \ldots, \mathrm{m}=1$ : i.e., azimuthal harmonics for low radial mode numbers) of large $(>200 \mu \mathrm{m})$ and the thickest $(75 \mathrm{~nm}$ thick) resonators (Figure 3(a)) shows a strong reduction to reach a minimum in dissipation. Further addition of azimuthal mode number results in marginal increase in dissipation. Hence for a given device size, dissipation reaches a minimum value, we define as dissipation floor indicated by dashed lines for 2 resonators in Figure 3. Similarly dissipation observed in higher azimuthal and radial harmonics $(\mathrm{n}>2, \mathrm{~m}>1)$ is also limited by this dissipation floor. We find that the pure radial modes of large resonators $(\mathrm{D}>200 \mu \mathrm{m})$ show a reduction in dissipation for higher radial harmonics $(\mathrm{n}=0, \mathrm{~m}=1,2,3, \ldots$,$) . Hence modal$ dependence of dissipation within an individual family of modes, $(\mathrm{n}=0,1,2, \mathrm{~m}=$ constant $)$ is greatly reduced for higher order radial modes and their azimuthal harmonics (not shown here) of large resonators $(>200 \mu \mathrm{m})$. These behaviors of higher order radial modes $(m>1)$ and their azimuthal harmonics are similar to those observed for optically defined resonators with non uniform cross section (average thickness $\sim 27 \mathrm{~nm})^{11}$ and will not be discussed further. As seen in Figure 3, limiting value of dissipation or the dissipation floor increases with the reduction in the diameter of the resonator. For smaller resonators, all radial modes (including 

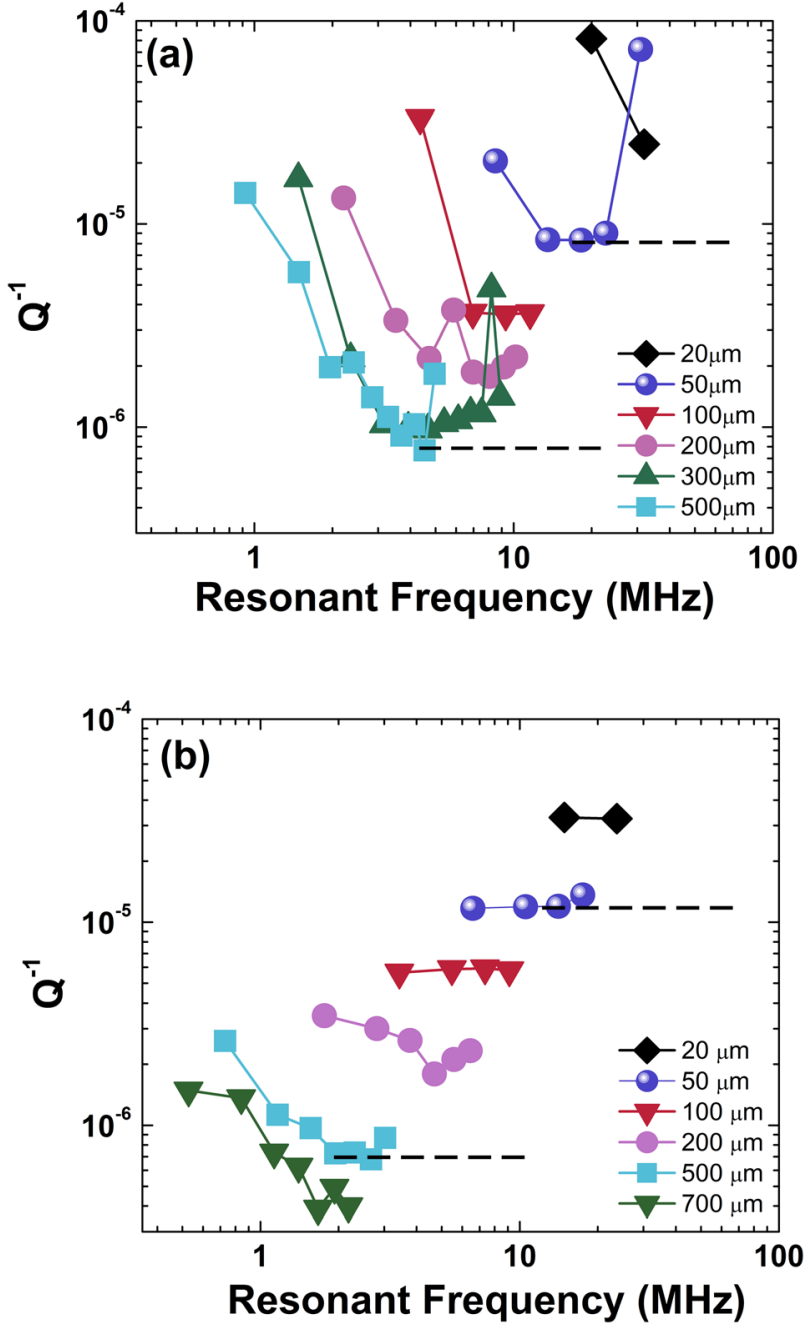

FIG. 3. Modal dependence of dissipation $\left(\mathrm{Q}^{-1}\right)$ for fundamental mode and its azimuthal harmonics $(\mathrm{n}=0,1,2,3, \ldots, \mathrm{m}=1)$ as a function of size and thickness of the resonator. (a) $75 \mathrm{~nm}$ thick e beam defined membranes clearly show a dissipation dominated by clamping loss for fundamental (radial) modes with the dissipation sharply decreasing as the number of azimuthal nodes increases before reaching a dissipation floor (represented by dashed lines for two resonators). At higher azimuthal harmonic numbers the dissipation gradually starts to increase above the dissipation floor. Thus we see more than an order of magnitude decrease in the dissipation with mode number for the largest membranes. In contrast the $\mathrm{Q}^{-1}$ of all modes of the thinner resonators ( $15 \mathrm{~nm}$ thick) shown in (b) more closely follows the dissipation floor illustrated by dashed lines which strongly depend on the size of the resonator. The lines shown join together points obtained from the same device for different modes.

the fundamental radial mode) and their azimuthal harmonics show a much reduced modal dependence of dissipation within a family of modes.

The dissipation of the fundamental mode $(0,1)$ is particularly important since it is most sensitive to clamping loss, ${ }^{11,12}$ and shows thickness dependence: thinner devices always show a lower dissipation. More interestingly, as the thickness of the resonators is decreased we observe a reduction in the modal dependence of $\mathrm{Q}^{-1}$ for all the modes within the individual modal families $(\mathrm{n}=0,1,2,3 \ldots$, for fixed low m) even for larger resonators ( $>200 \mu \mathrm{m}$ ) as shown in Figure 3(b). This indicates that the dissipation mechanism governing the fundamental mode and lower order azimuthal harmonics $(\mathrm{n}=0,1,2, \mathrm{~m}=1)$ is strongly influenced by the thickness of the resonator. For thinner $(15 \mathrm{~nm})$ e-beam defined devices, the $\mathrm{Q}$ of the pure radial modes including the fundamental mode $(\mathrm{n}=0, \mathrm{~m}=1,2)$ increased by an order of magnitude for resonators of large diameters (D $>200 \mu \mathrm{m}$ ) and a quality factor of upto $4.4 \times 10^{6}$ has been observed in a higher order radial mode $(\mathrm{n}=0, \mathrm{~m}=5)$ for a $700 \mu \mathrm{m}$ drum resonator (Figure 2 inset). In Figure 4(a), we compare the dissipation measured for large ( $300 \mu \mathrm{m}$ to $500 \mu \mathrm{m}$ diameter) and small (50 $\mu \mathrm{m}$ diameter) resonators as a function of thickness. We observe that for a given device, maximum achievable $\mathrm{Q}$ is increases linearly with the diameter of the resonator. In other words dissipation floor decreases linearly with increase in diameter and the thickness dependence is marginal (Figure 4(b)). It is evident that the dissipation floor is
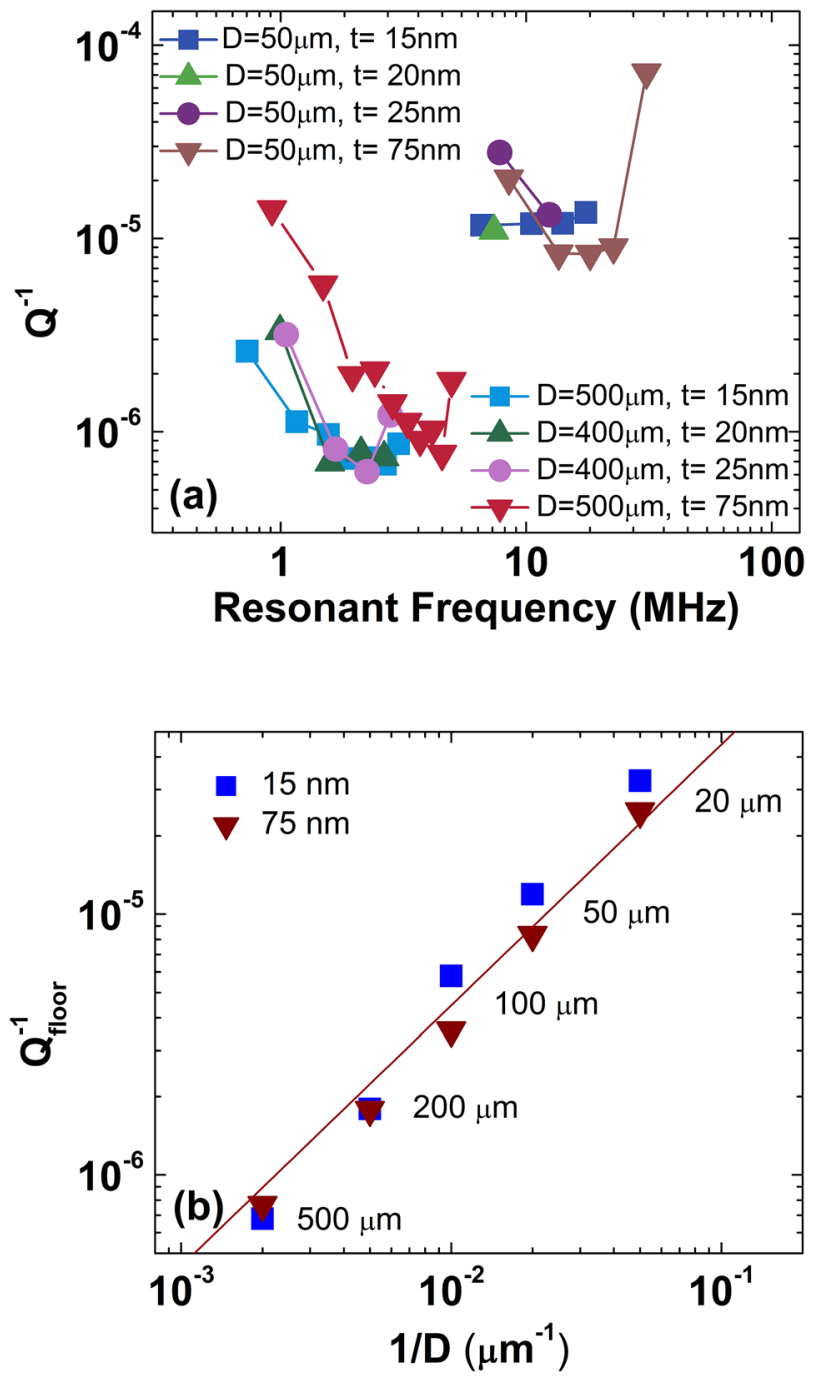

FIG. 4. Thickness dependence of dissipation $\left(\mathrm{Q}^{-1}\right)$ of fundamental and subsequent higher order azimuthal modes $(n=0,1,2,3, \ldots, m=1)$ of resonators of comparable dimensions. (a) The fundamental mode exhibits thickness dependence, with thinner resonators presenting higher Q's. The Q of thinner membranes $\left(\mathrm{t} \leq 25 \mathrm{~nm}\right.$, initial film thickness $\left.t_{\mathrm{i}}=55 \mathrm{~nm}\right)$ is relatively mode independent and is limited by an intrinsic dissipation mechanism. Dissipation floor scales inversely with the diameter of the membrane, and is weakly thickness dependent, with thinner resonators showing higher Q's (b) for large resonators ( $\mathrm{D}>200 \mu \mathrm{m}$ ). However for smaller resonators this dissipation trend is not observed. The solid line in (b) shows the best fit to both data sets with $\mathrm{Q}^{-1} \alpha \mathrm{D}^{-1}$ 
slightly lower for the thick resonators with the exception of the large diameter (D $>200 \mu \mathrm{m})$ devices.

Dissipation in these high stress string and membrane resonators has been discussed in previous articles. ${ }^{3,4,6,12,15-17}$ Overall, the measured dissipation of these high tensile stress membranes and strings is strongly dependent on the size and the mode type of the resonator. ${ }^{3,4,6,12}$ This behavior has been attributed both to the curvature dependence of the internal dissipation ${ }^{3,16,17}$ and to clamping (or support) losses. ${ }^{11,12}$ For a given mode $(\mathrm{n}=0,1,2 \ldots, \mathrm{m}=1,2,3$.$) , the dissipation due$ to clamping loss of a membrane resonator is predicted to be inversely proportional to the aspect ratio $(\mathrm{D} / \mathrm{t})^{12}$

$$
\frac{1}{Q_{\text {clamping }}}=A(n, m) \sigma^{\frac{3}{2}} \frac{t}{D} \text {. }
$$

Here $t$ is the thickness of the resonator. A is the prefactor associated with clamping loss that depends on the mode number of the resonator. For higher order azimuthal modes $(\mathrm{m}=$ constant $)$ of the high stress drums, a drastic reduction in dissipation within the individual radial mode "family" $\left(\omega_{\mathrm{n}=0,1,2, \ldots, \mathrm{m}}\right)$ is predicted due to destructive interference between the elastic waves radiated from adjacent equivalent segments of the resonator as the azimuthal harmonic numbers increase. ${ }^{12} \mathrm{We}$ experimentally observe this increase in the quality factor (Figure 3(a)) within the individual modal family for large drums $(\mathrm{D}>200 \mu \mathrm{m})$ made from thicker films $(75 \mathrm{~nm})$. Importantly, we observe a nearly linear dependence of dissipation with inverse aspect ratio (t/D) for the fundamental radial mode (dominated by clamping losses) as shown in Figure 5(a), consistent with the dependence described by Eq. (2). However, the observed dissipation "floor" in e-beam or optically defined nitride membranes cannot be explained by the clamping loss model. ${ }^{11,12}$ Observed dissipation in high stress nitride membranes could be a combination of clamping losses (limits the maximum achievable Q for fundamental mode) and internal losses (limits the maximum $\mathrm{Q}$ that can be reached for a given device size). Further evidence for this internal dissipation mechanism can be seen in ultra thin membranes where modal dependence of dissipation predicted by the clamping loss model (Eq. (2)) is suppressed, and replaced by a diameter dependent dissipation floor (Figure 4(b)). The dissipation in turn gradually increases for higher azimuthal mode numbers (for a given radial mode number) indicating the weak modal dependence shown by internal dissipation mechanism.

The internal dissipation mechanism in high-stress resonators has been modeled recently, ${ }^{3,17}$ where an increase in $\mathrm{Q}$ compared to stress-free resonators $\left(\mathrm{Q}_{\text {intrinsic },(\sigma=0)}\right)$ has been attributed to an enhancement of the stored energy in the resonator. The observed internal dissipation in these high-stress resonators depends on the aspect ratio and the mode type, a dependency that is linked to the enhanced local strain associated to the clamping points and the antinodes of the resonator eigenmodes. The increased curvature at the latter accounts for the increased dissipation exhibited by the higher order modes, while the higher dissipation observed in small resonators (for all the modes compared to large resonators)
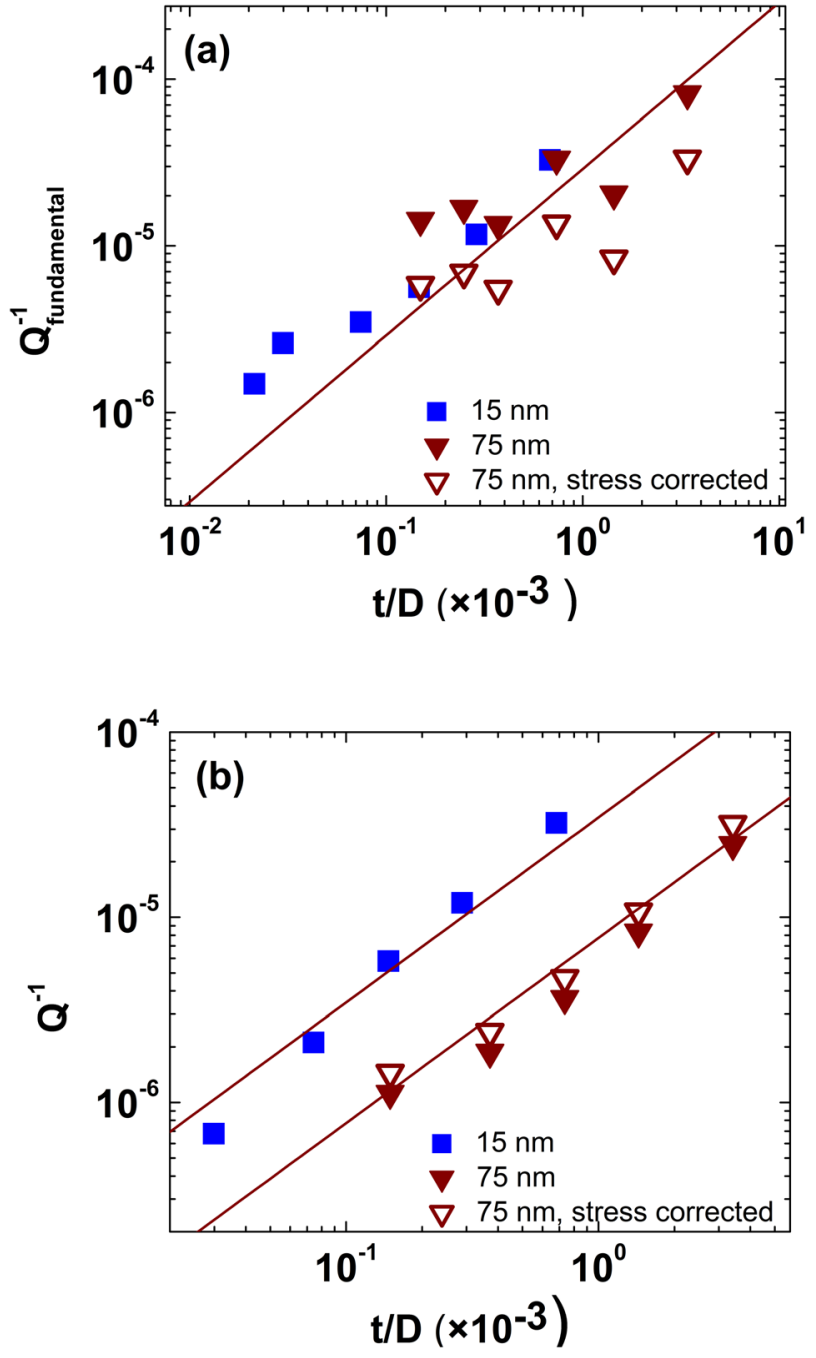

FIG. 5. Dissipation as a function of inverse aspect ratio (thickness to diameter or $t / D$ ) of the silicon nitride membrane resonators. The open triangles represent the correction to the dissipation of the $75 \mathrm{~nm}$ thick resonators if they were to have the lower average stress seen in the $15 \mathrm{~nm}$ thick resonators. In (a) we plot the fundamental mode $(0,1)$ of various diameter resonators having two thicknesses. These two data sets follow the same trend as shown by the solid line introduced to illustrate the $\mathrm{Q}^{-1}$ varying as $t / D$, consistent with the clamping loss model (Eqs. (2) and (3)). In (b) we plot the dissipation $\left(\mathrm{Q}^{-1}\right)$ of the representative modes that are dominated by internal dissipation mechanism. Representative data (taken from Figure 3) are shown for diameters $500 \mu \mathrm{m}(\mathrm{n}=5, \mathrm{~m}=1), 200 \mu \mathrm{m}(4,1), 100 \mu \mathrm{m}(3,1)$, $50 \mu \mathrm{m}(2,1)$, and $20 \mu \mathrm{m}(1,1)$. The dissipation floor fails to scale as t/D.

results from the relative importance of the contribution associated with the clamping points. Hence the overall dissipation in high stress membrane resonators can be written as,

$$
\begin{aligned}
Q^{-1}= & Q_{\text {clamping }}^{-1}+Q_{\text {int,clamps }}^{-1}+Q_{\text {int,antinodes }}^{-1} \\
Q^{-1}= & A(n, m) \sigma^{\frac{3}{2}}\left(\frac{t}{D}\right)+Q_{\text {intrinsic, },(\sigma=0)}^{-1} \\
& \times\left(B \frac{1}{\sqrt{\sigma}}\left(\frac{t}{D}\right)+C(n, m) \frac{1}{\sigma}\left(\frac{t}{D}\right)^{2}\right),
\end{aligned}
$$

where $\mathrm{B}$ and $\mathrm{C}$ are the prefactors corresponding to the internal losses associated with the clamps and antinodes (we note that the latter contribution is not localized at the antinodes but rather distributed over the "bulk" of the resonator). ${ }^{18}$ 
Figure 5(a) shows the dissipation of the fundamental mode (dominated by clamping losses); Figure 5(b) shows the dissipation of a representative higher order azimuthal mode dominated by internal dissipation as a function of the inverse aspect ratio (t/D) of the resonator. While modes dominated by clamping losses exhibit an approximately linear scaling with t/D across both thicknesses (Figure 5(a)), the dissipation of higher order modes (dominated by internal mechanism) which also scale linearly, do not fall on the same line for two different thicknesses (Figure 5(b)). The third term in Eq. (3), invoked to account for the mode dependent internal dissipation at high mode numbers should scale as $(\mathrm{t} / \mathrm{D})^{2}$ and evidently does not contribute to the dissipation floor.

Stress also affects the dissipation and could account for the improper scaling of the data from the two different thickness resonators. Equation (3) predicts that clamping losses increase with tensile stress $(\sigma)$, while intrinsic losses decrease with increased stress. In Figure 5, we scale the dissipation observed in $75 \mathrm{~nm}$ resonators to account for the reduced effective stress (inferred from Figure 2) in the 15 $\mathrm{nm}$ thick resonator. In Figure 5(a), we see that the scaled dissipation (obtained from the first term of Eq. (3)) leads to a decreased clamping loss term. However, the general trend of $\mathrm{Q}^{-1}$ varying as $\mathrm{t} / \mathrm{D}$ is preserved. After correcting for the change in stress in the second term of Eq. (3), the $\mathrm{Q}^{-1}$ vs. inverse aspect ratio is only marginally altered as shown in Figure 5(b). Thus, for same diameter and mode number, the scaled dissipation (dominated by internal dissipation) of the $75 \mathrm{~nm}$ thick membranes does not follow the dissipation of the $15 \mathrm{~nm}$ thick membrane. The discrepancy in the scaling could be due to the presence of an internal dissipation mechanism which is unrelated to the thickness of the resonator (which follows by comparing Figures 5(b) and 4(b)), unless the $\mathrm{Q}_{\text {intrinsic, }(\sigma=0)}$ term in Eq. (3) follows a linear thickness dependence. To summarize we find that the dissipation floor varies as $\mathrm{D}^{-1}$ (Figure $4(\mathrm{~b})$ ), but is not consistent with the $\mathrm{t} / \mathrm{D}$ dependence described in Eq. (3), since the dissipations of the $15 \mathrm{~nm}$ and $75 \mathrm{~nm}$ membranes do not collapse onto a single line (Figure 5(b)).

It has been previously observed that dissipation in mechanical resonators is volume dependent. ${ }^{9}$ Resonators show an increase in dissipation with reduction in volume and this has been mainly attributed to the dissipation mechanisms associated with the surface. ${ }^{9,10}$ As the membranes are thinned, the increased surface to volume ratio $(\mathrm{R})$ results in dissipation mechanisms that are primarily related to stress assisted relaxation of defects, dangling bonds or contamination at the surface. To illustrate how distinctly the resonators described in this paper behave when compared to previously investigated structures, we examine in Figure 6 the volume to surface ratio $(\sim t)$ versus $Q$ of various resonators made from different materials ${ }^{4,9,10,19}$ and tensioned silicon nitride membranes of different thickness. The high stress silicon nitride membranes, show very high $\mathrm{Q}$ for very high surface to volume ratio $(\mathrm{R})$, with the highest measured $\mathrm{Q}$ increasing as the thickness is reduced, contrary to the trend observed in other systems. ${ }^{10}$ The highest measured quality factor for our resonators is for a radial mode $\left(\mathrm{Q}=4.4 \times 10^{6}\right)$ of a $700 \mu \mathrm{m}$ diameter, $15 \mathrm{~nm}$ thick resonator with very high surface to volume

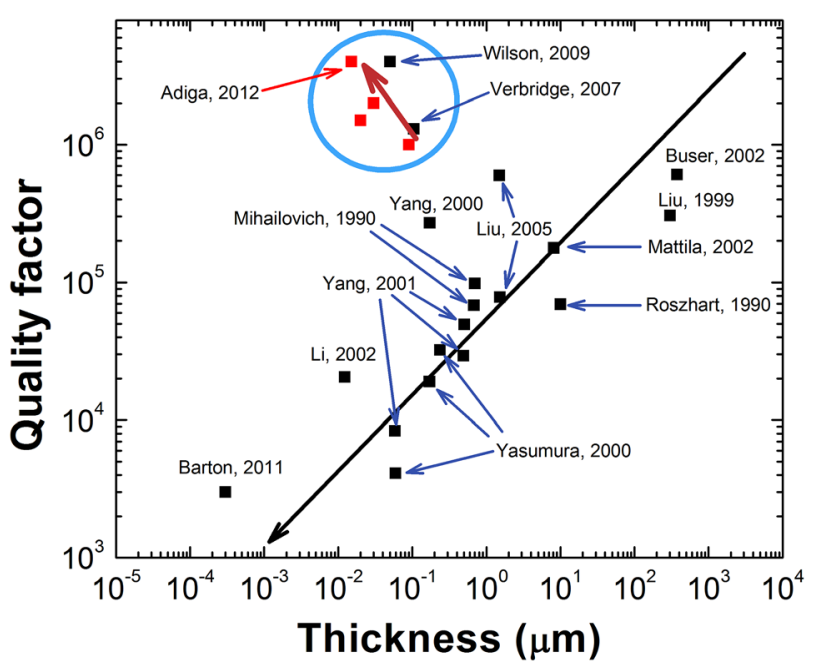

FIG. 6. Thickness dependence of quality factors of different resonators studied in the literature ${ }^{2,6,10,19}$ compared to the resonators measured for this study displaying the highest Q's measured for a given thickness (red squares). These show an increase in $\mathrm{Q}$ with reduced thickness (i.e., surface to volume ratio, $\mathrm{R}$ ) indicating that ultrathin silicon nitride resonators can achieve high quality factors with an appropriate combination of tensile stress, size, and modes of the resonators.

ratio $\left(\mathrm{R}=0.13 \mathrm{~nm}^{-1}\right)$ that yields an $R Q \sim 600000 \mathrm{~nm}^{-1}$. Such a resonator offers a unique potential for mass or force sensors. Silicon nitride resonators are actively pursued for optomechanical experiments with the possibility of using ultra thin nitride membrane resonators in high finesse cavities, ${ }^{8}$ where high mechanical Q, low mass and low spring constants are desirable. In this regard, large ultrathin resonators with high quality factors as fabricated here, provide a useful means to achieve and explore the interaction between optical and mechanical degrees of freedom.

\section{CONCLUSIONS}

In conclusion, we have measured the mechanical dissipation in ultra thin circular silicon nitride membranes as a function of size, mode, shape, and thickness. The observed $\mathrm{Q}^{-1}$ of the resonators is strongly mode dependent for thicker films due to clamping loss. Clamping loss of resonators can be minimized by increasing the aspect ratio of the resonators $(\mathrm{D} /$ t). For thinner membranes, the observed quality factors are limited by an intrinsic dissipation mechanism. The highest achievable quality factors for ultrathin silicon nitride membranes scales linearly with the diameter, a dependence also seen in self tensioned graphene drums. ${ }^{19}$ We observe a weak thickness dependent $\mathrm{Q}^{-1}$ floor for these membrane resonators, resulting in a higher RQ product for thinner membranes. This indicates that two dimensional materials like graphene ${ }^{19}$ and ultrathin silicon nitride (this study) can achieve high quality factors by an appropriate combination of tensile stress, size, and mode of the resonators enabling the use of such devices for optomechanical experiments and resonant sensors.

\section{ACKNOWLEDGMENTS}

We thank the support from Cornell Nanofabrication Facility and acknowledge financial support from NSF grants 
DMR-0908634, ECCS-1001742, and DMR 1120296. I.W.R. acknowledges the financial support from DFG grant WI$3859 / 1-1$.

${ }^{1}$ S. S. Verbridge, H. G. Craighead, and J. M. Parpia, Appl. Phys. Lett. 92, 013112 (2008).

${ }^{2}$ D. J. Wilson, C. A. Regal, S. B. Papp, and H. J. Kimble, Phys. Rev. Lett. 103, 207204 (2009)

${ }^{3}$ Q. P. Unterreithmeier, T. Faust, and J. P. Kotthaus, Phys. Rev. Lett. 105, 027205 (2010).

${ }^{4}$ S. S. Verbridge, J. M. Parpia, R. B. Reichenbach, L. M. Bellan, and H. G. Craighead, J. Appl. Phys. 99, 124304 (2006).

${ }^{5}$ B. M. Zwickl, W. E. Shanks, A. M. Jayich, C. Yang, A. C. Bleszynski Jayich, J. D. Thompson, and J. G. E. Harris, Appl. Phys. Lett. 92, 103125 (2008).

${ }^{6}$ S. S. Verbridge, D. F. Shapiro, H. G. Craighead, and J. M. Parpia, Nano Lett. 7, 1728 (2007).

${ }^{7}$ S. Groblacher, J. B. Hertzberg, M. R. Vanner, G. D. Cole, S. Gigan, K. C. Schwab, and M. Aspelmeyer, Nat. Phys. 5, 485 (2009).

${ }^{8}$ J. D. Thompson, B. M. Zwickl, A. M. Jayich, F. Marquardt, S. M. Girvin, and J. G. E. Harris, Nature (London) 452, 72 (2008).
${ }^{9}$ P. Mohanty, D. A. Harrington, K. L. Ekinci, Y. T. Yang, M. J. Murphy, and M. L. Roukes, Phys. Rev. B 66, 085416 (2002).

${ }^{10}$ X. Liu, J. F. Vignola, H. J. Simpson, B. R. Lemon, B. H. Houston, and D. M. Photiadis, J. Appl. Phys. 97, 023524 (2005).

${ }^{11}$ V. P. Adiga, B. Ilic, R. A. Barton, I. Wilson-Rae, H. G. Craighead, and J. M. Parpia, Appl.Phys. Lett. 99, 253103 (2011).

${ }^{12}$ I. Wilson-Rae, R. A. Barton, S. S. Verbridge, D. R. Southworth, B. Ilic, H. G. Craighead, and J. M. Parpia, Phys. Rev. Lett. 106, 047205 (2011).

${ }^{13}$ B. Ilic, S. Krylov, K. Aubin, R. Reichenbach, and H. G. Craighead, Appl. Phys. Lett. 86, 193114 (2005).

${ }^{14}$ K. F. Graff, Wave Motion in Elastic Solids (Dover Publications, 1991).

${ }^{15}$ D. R. Southworth, R. A. Barton, S. S. Verbridge, B. Ilic, A. D. Fefferman, H. G. Craighead, and J. M. Parpia, Phys. Rev. Lett. 102, 225503 (2009).

${ }^{16}$ S. Schmid, K. D. Jensen, K. H. Nielsen, and A. Boisen, Phys. Rev. B 84, 165307 (2011).

${ }^{17}$ P.-L. Yu, T. P. Purdy, and C. A. Regal, Phys. Rev. Lett. 108, 083603 (2012).

${ }^{18}$ Contribution of internal dissipation in Eq. (3) is modified from the one developed for square resonators in Ref. 17. We have replaced the lateral dimension $\mathrm{L}$, by the diameter $\mathrm{D}$.

${ }^{19}$ R. A. Barton, B. Ilic, A. M. van der Zande, W. S. Whitney, P. L. McEuen, J. M. Parpia, and H. G. Craighead, Nano Lett. 11, 1232 (2011). 